\title{
Properties of Non-differentiating Derivatives of Streptomyces hygroscopicus
}

\author{
By MARTIN ROTH, * DIETER NOACK AND GÜNTHER REINHARDT \\ Akademie der Wissenschaften der DDR, Forschungszentrum für Molekularbiologie und Medizin, \\ Zentralinstitut für Mikrobiologie und experimentelle Therapie, Beutenbergstrasse 11, \\ P.O. Box 73, DDR-6900 Jena, German Democratic Republic
}

(Received 16 July 1981; revised 8 November 1981)

\begin{abstract}
The properties of non-differentiating derivatives of Streptomyces hygroscopicus, which appeared spontaneously in continuous cultures of their parental strain, were investigated. These derivatives differ from the original strain by production of only trace amounts of the antibiotic turimycin, inability to form aerial mycelium and spores, and altered morphology of colonies and submerged mycelium. It is proposed that as a consequence of a genetic alteration the derivatives are impaired in a central regulatory function influencing differentiation processes by pleiotropic effects.
\end{abstract}

\section{INTRODUCTION}

During continuous culture of Streptomyces hygroscopicus, derivatives appeared which were no longer able to form the macrolide antibiotic turimycin $\left(\mathrm{Tur}^{-}\right)$and aerial mycelium $\left(\mathrm{Amy}^{-}\right)$. Because these non-differentiating derivatives failed to revert to the original phenotype $\left(\mathrm{Tur}^{+} \mathrm{Amy}^{+}\right.$), it was suggested that their occurrence was the consequence of a genetic alteration, possibly the loss of extrachromosomal DNA (Roth \& Noack, 1982).

In the present communication we report more detailed studies of the properties of the Tur $^{-}$Amy $^{-}$clones in comparison with the parental strain. Our aim was to obtain information about the reason for the inability of these clones to differentiate.

\section{METHODS}

Bacterial strains. The mutant NG60-93 of Streptomyces hygroscopicus JA6599 was described recently. Nondifferentiating derivatives of the strain appeared at high frequency during continuous culture at $30^{\circ} \mathrm{C}$ (Roth \& Noack, 1982). Five of these derivatives were used in this study: $\mathrm{CC} 1$ and $\mathrm{CC} 292$ were isolated from glucoselimited chemostat cultures grown at a dilution rate of $0 \cdot 1 \mathrm{~h}^{-1}: \mathrm{CC} 335, \mathrm{CC} 382$ and CC415 were obtained from ammonium chloride-limited cultures grown at a dilution rate of $0 \cdot 3 \mathrm{~h}^{-1}$. Agar slant cultures of the strains were prepared as described previously (Roth \& Noack, 1982) except that non-differentiating strains were incubated for $5 \mathrm{~d}$ only. Bacillus subtilis ATCC 6633 was used as the test organism in the bioassay for turimycin.

Media. Propagation of the S. hygroscopicus strains and platings were done on AL53 agar (Roth \& Noack, 1982). Submerged cultures were grown in liquid AL53 medium or in mineral salts medium (Roth \& Noack, 1982)

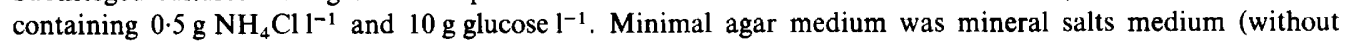
Casamino acids) supplemented with $15 \mathrm{~g}$ agar $1^{-1}$.

Detection of turimycin biosynthesis in liquid medium. AL53 medium $(80 \mathrm{ml}$ in $500 \mathrm{ml}$ glass flasks) was inoculated with spore or mycelium suspensions and incubated on a rotary shaker $\left(240 \mathrm{rev}\right.$. $\mathrm{min}^{-1}, 5 \mathrm{~cm}$ stroke $)$ at $28^{\circ} \mathrm{C}$. The turimycin content in culture filtrates was determined by means of an agar diffusion bioassay using $B$. subtilis as the test organism. For chromatography, 31 of culture filtrate of each Tur $^{-}$Amy $^{-}$strain was extracted three times with $300 \mathrm{ml}$ chloroform, and the combined extracts were evaporated to $10 \mathrm{ml}$. Thin-layer chromatography to detect turimycin was performed according to Fricke et al. (1978).

Sensitivity to turimycin. The sensitivity of the $S$. hygroscopicus strains in relation to the growth phase of the cultures was tested by plating appropriate dilutions of the culture on AL53 agar plates containing different concentrations of turimycin. Colonies were counted after $6 \mathrm{~d}$ incubation at $28^{\circ} \mathrm{C}$. The effect of the antibiotic on exponentially growing mycelium was studied by adding different concentrations of turimycin to cultures in the early-exponential phase of growth. Cultures were grown in $50 \mathrm{ml}$ mineral salts medium in $450 \mathrm{ml}$ Fernbach flasks 
shaken on a reciprocal shaker (frequency $130 \mathrm{rev} \cdot \mathrm{min}^{-1}$ ). Growth was followed by measurement of turbidity at $470 \mathrm{~nm}$.

\section{RESULTS}

\section{Turimycin biosynthesis in liquid medium}

The non-differentiating derivatives, which are unable to produce turimycin on agar medium (Roth \& Noack, 1982), were tested for antibiotic biosynthesis in liquid complex medium. Cultures were grown for $120 \mathrm{~h}$, and samples were withdrawn at $24 \mathrm{~h}$ intervals. In cultures of the Tur $^{+}$strain NG60-93, the maximum turimycin concentration of $1200 \mathrm{U} \mathrm{ml}^{-1}(1 \mathrm{U}=0.75 \mu \mathrm{g})$ was found $48 \mathrm{~h}$ after inoculation. In cultures of all Tur ${ }^{-}$clones, turimycin was not detected either by the agar diffusion bioassay or by chromatography. However, after extraction of culture filtrates $(72 \mathrm{~h})$ with chloroform, which resulted in a 300 -fold concentration, trace amounts of antibiotic (about $2 \mu \mathrm{g} \mathrm{ml}^{-1}$ ) were detected chromatographically. The proportions of the different turimycin components were the same as those produced by strain NG60-93. Abnormal concentrations of turimycin precursors, e.g. platenolides I and II (Gräfe et al., 1980), were not observed.

\section{Sensitivity to turimycin}

Antibiotic-producing micro-organisms develop resistance to high concentrations of their own antibiotic by different mechanisms (for review, see Demain, 1974). Therefore, we investigated whether the inability of the Tur $^{-}$Amy $^{-}$strains to produce turimycin was connected with an altered susceptibility to the antibiotic. In preliminary experiments we found that none of the

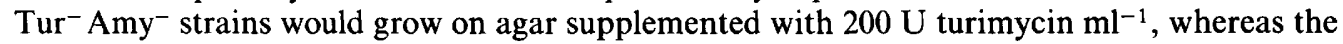
$\mathrm{Tur}^{+}$strain NG60-93 tolerated $1000 \mathrm{U} \mathrm{ml}^{-1}$. More detailed comparative studies were therefore carried out with strains $\mathrm{CC} 1$ and NG60-93.

The turimycin resistance of strain NG60-93 depended on the growth phase of the organism (Fig. 1a). Spores were resistant to turimycin up to $200 \mathrm{U} \mathrm{ml}^{-1}$, whereas mycelium in the earlyexponential phase of growth ( $8 \mathrm{~h}$ after inoculation) was sensitive to low concentrations of the antibiotic. Colony formation from mycelium in the turimycin production phase ( $24 \mathrm{~h}$ and later) was not impaired by antibiotic concentrations up to $1000 \mathrm{U} \mathrm{ml}^{-1}$. In contrast, strain $\mathrm{CCl}$ was sensitive to turimycin irrespective of the growth phase (Fig. $1 b$ ). The colony-forming ability of mycelium in the early-exponential phase of growth was most strongly inhibited by the antibiotic. On agar containing turimycin at $10 \mathrm{U} \mathrm{ml}^{-1}$, colony formation was three orders of magnitude lower than on antibiotic-free medium. The colonies growing at 50 and $100 \mathrm{U}$ turimycin ml-1 remained small. No growth was observed on agar supplemented with $200 \mathrm{U} \mathrm{ml}^{-1}$.

The sensitivity to turimycin of cultures of both strains in the exponential phase of growth was investigated in more detail (Fig. 2). After addition of 20 or $200 \mathrm{U}_{\text {turimycin ml }}^{-1}$ to growing cultures of each strain, growth continued for about half an hour. Thereafter growth stopped, caused by inhibition of protein synthesis (Haupt et al., 1976). Strain NG60-93 was able to overcome this inhibition after $1 \mathrm{~h}$ with $20 \mathrm{U}$ turimycin $\mathrm{ml}^{-1}$ and after $20 \mathrm{~h}$ with $200 \mathrm{U}$ turimycin $\mathrm{ml}^{-1}$ (Fig. 2a). The period of growth inhibition depended on the mycelium concentration in the culture and was shorter with increased biomass. The growth rate in turimycin-containing medium was reduced. Growth of strain $\mathrm{CCl}$ in medium containing $20 \mathrm{U}$ turimycin $\mathrm{ml}^{-1}$ occurred only after an extended lag and at a reduced rate (Fig. $2 b$ ). At $200 \mathrm{U} \mathrm{ml}^{-1}$ the mycelium died.

\section{Growth properties}

Besides their inability to form turimycin and aerial mycelium, the $\mathrm{Tur}^{-} \mathrm{Amy}^{-}$derivatives were distinguished from the parental strain by an altered colony morphology. Old colonies of all non-differentiating mutants had an irregular folded surface and a soft consistency, caused by fragmentation and lysis of the mycelium. In contrast, differentiated colonies of NG60-93 were compact and had an even surface apart from some radial folds. In submerged cultures of Turderivatives, growth was prolonged compared with that of the parental strain, resulting in 


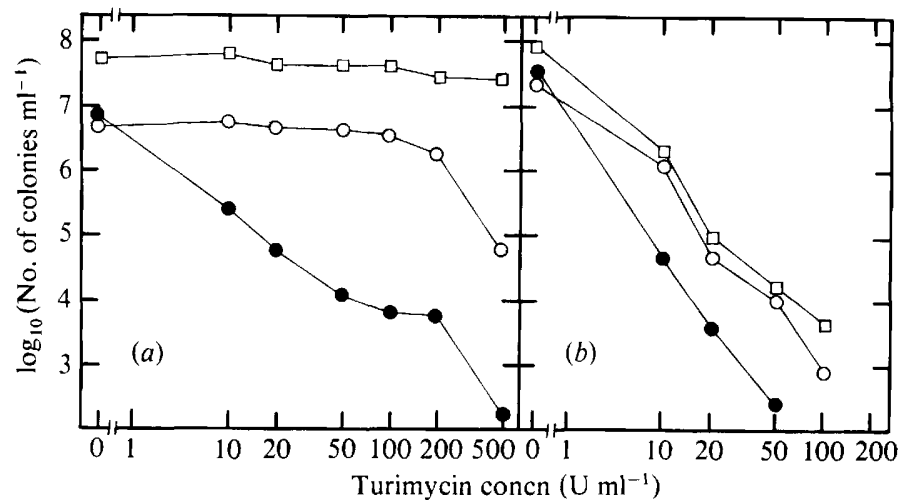

Fig. 1. Susceptibility to turimycin of S. hygroscopicus NG60-93 (a) and its non-differentiating derivative $\mathrm{CCl}(b)$ in relation to the growth phase. Samples taken from submerged cultures of the strains immediately after inoculation $(\mathrm{O})$ with spores (NG60-93) or mycelial pieces (CCl) from agar slants, or $8 \mathrm{~h}(\mathrm{O})$ and $24 \mathrm{~h}(\square)$ after inoculation, were plated on agar medium containing different amounts of turimycin. After $6 \mathrm{~d}$ incubation the numbers of colonies per $\mathrm{ml}$ were determined.

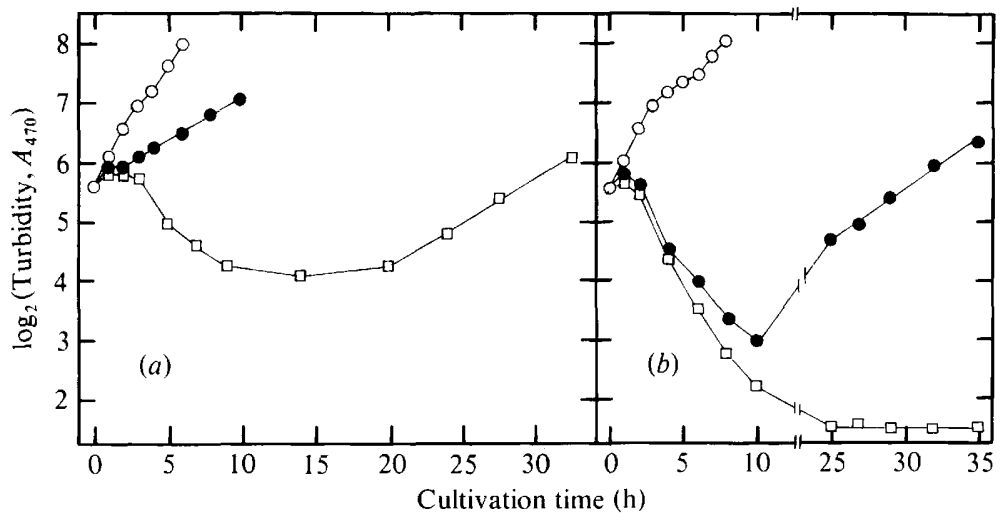

Fig. 2. Effect of turimycin on exponentially growing cultures of $S$. hygroscopicus NG60-93 (a) and its non-differentiating derivative $\mathrm{CCl}(b)$. Turimycin was added to submerged cultures in the earlyexponential phase of growth at time 0 [control without turimycin $(O) ; 20 \mathrm{U} \mathrm{ml}^{-1}(\Theta) ; 200 \mathrm{U} \mathrm{ml}^{-1}$ (口)]. Growth was followed by measurement of turbidity at $470 \mathrm{~nm}$.

increased biomass production. After about $48 \mathrm{~h}$ cultivation the mycelium began to break into rod-like pieces of different size. These mycelial fragments failed to survive more than $2 \mathrm{~d}$ after the end of growth in the submerged culture. Thus, they cannot be regarded as persisting forms or spores. Mycelial fragmentation in submerged cultures of strain NG60-93 did not occur.

Both strain NG60-93 and the non-differentiating derivatives were prototrophic. Loss of differentiation functions connected with arginine auxotrophy as reported by Redshaw et al. (1979) and Nakano \& Ogawara (1980) was not observed.

The turimycin-producing $S$. hygroscopicus is lysogenic for the species-specific temperate actinophage SH3 (Rautenstein et al., 1976) and resistant to infection by this phage (Roth et al., 1978). The Tur $^{-} \mathrm{Amy}^{-}$mutants were not altered in these two characters.

\section{Attempts to detect a bioregulator effecting differentiation in $S$. hygroscopicus}

Bioregulators or effectors inducing or stimulating differentiation processes such as antibiotic, aerial mycelium and spore formation have been found in various streptomycetes (Khokhlov et al., 1973; McCann \& Pogell, 1979; Biro et al., 1980). Therefore, we tested whether the inability of Tur $^{-}$Amy $^{-}$strains of $S$. hygroscopicus to differentiate is caused by the inability to form a bioregulator. The non-differentiating strains and strain NG60-93 were inoculated on AL53 agar in strips side by side without direct contact. A diffusible bioregulator excreted by the differen- 
tiating strain should stimulate antibiotic and aerial mycelium formation in the Tur ${ }^{-}$Amy $^{-}$ derivatives. However, neither turimycin production, assayed by overlaying with soft agar containing $B$. subtilis, nor aerial mycelium formation were induced in the non-differentiating derivatives. Furthermore, when mycelium of the $\mathrm{Tur}^{-} \mathrm{Amy}^{-}$strains from submerged cultures, used for detection of turimycin biosynthesis, was resuspended in culture filtrates of the parental $\mathrm{Tur}^{+} \mathrm{Amy}^{+}$strain from different growth phases, during subsequent cultivation no turimycin formation by the non-differentiating derivatives was observed. In those cases where the Tur $^{-} \mathrm{Amy}^{-}$mycelium was inoculated into culture filtrates containing more than $200 \mathrm{U}$ turimycin $\mathrm{ml}^{-1}$ further growth did not occur.

From these findings we conclude that the Tur ${ }^{-} \mathrm{Amy}^{-}$derivatives cannot be stimulated to differentiate by any diffusible metabolic product excreted from the parental $\mathrm{Tur}^{+} \mathrm{Amy}^{+}$strain.

\section{DISCUSSION}

The strains of $S$. hygroscopicus investigated in this study differ from the parental strain in several differentiated functions including turimycin production, susceptibility to turimycin, capacity to form aerial mycelium and spores, colony morphology and mycelial morphology in submerged cultures. In view of the spontaneous appearance of these strains without mutagenic treatment and their inability to revert to the original phenotype (Roth \& Noack, 1982), it is unlikely that the altered characters result from single mutations. More probably, the nondifferentiating strains are impaired in a central regulatory function which influences differentiation processes by pleiotropic effects. This suggestion is supported by the following experimental data. (i) The structural genes for turimycin biosynthesis seem to be undamaged in the Tur derivatives, as indicated by the detection of trace amounts of all turimycin components that are produced by the $\mathrm{Tur}^{+}$strain. Accumulation of turimycin precursors, which could result from a mutation in a structural gene for turimycin biosynthesis, was not found. (ii) Fragmentation of the mycelium which may be involved in spore formation was observed in submerged and surface cultures of the Tur $^{-}$Amy $^{-}$derivatives after the exponential phase of growth. However, regulated spore formation did not occur. (iii) The results of our experiments concerning turimycin resistance in $S$. hygroscopicus suggest a mechanism similar to that responsible for streptomycin resistance in S. griseus (Cella \& Vining, 1975). The induction of resistance to low concentrations of turimycin during the exponential phase of growth in both $\mathrm{Tur}^{+}$and $\mathrm{Tur}^{-}$strains might be explained by formation of an antibiotic-inactivating enzyme. However, this enzymic activity seems to be insufficient to protect the organism from high amounts of the antibiotic penetrating into the cells. Such high amounts would be present during the antibiotic production phase of the producing strain. In this case there may be a second resistance mechanism which alters the permeability barrier to decrease uptake of the antibiotic. Such a mechanism would require structural and functional alterations of the cell membrane. The differences in the susceptibility to turimycin between the non-differentiating strains and the parent organism may reflect that only the enzymic resistance mechanism acts in the $\mathrm{Tur}^{-} \mathrm{Amy}^{-}$clones.

Structural and functional alterations of the cell membrane and wall are necessary not only for developing antibiotic resistance but also for antibiotic production (e.g. for active transport of the drug out of the cells) and for morphological differentiation. The simultaneous loss of these differentiated functions in the $\mathrm{Tur}^{-} \mathrm{Amy}^{-}$derivatives and the prolonged growth compared with the parental strain point to impaired regulation of cell membrane and wall differentiation. This is supported by differences between $\mathrm{Tur}^{+}$and Tur $^{-}$strains with respect to fatty acid and lipid composition of the mycelia (Gräfe et al., 1982).

We thank Karin Gaube for skilful technical assistance.

\section{REFERENCES}

Biro, S., Bekesi, I., Vitalis, S. \& Szabo, G. (1980). A substance effecting differentiation in Streptomyces griseus. Purification and properties. European Journal of Biochemistry 103, 359-364.
Cella, R. \& Vining, L. C. (1975). Resistance to streptomycin in a producing strain of Streptomyces griseus. Canadian Journal of Microbiology 21, 463472 . 
Demain, A. L. (1974). How do antibiotic-producing microorganisms avoid suicide? Annals of the New York Academy of Sciences 235, 601-612.

Fricke, H., Mühlig, P. \& KüHNE, C. (1978). Quantitativ dünnschicht-chromatographische Bestimmung des Makrolidantibiotikums Turimycin $\mathrm{H}$ und einiger Abbauprodukte durch densitometrische in-situ Messung mit dem Bildanalysegerät "Quantimet 720". Journal of Chromatography 153, 495-506.

GräFe, U., Fleck, W. F., Schade, W., Reinhardt, G., Tresselt, D. \& ThruM, H. (1980). The platenolides I and II as precursors of turimycin. Journal of Antibiotics 33, 663-664.

GräFe, U., ReinhardT, G., Krebs, D., Roth, M. \& NoACK, D. (1982). Altered lipid composition in a non-differentiating derivative of Streptomyces hygroscopicus. Journal of General Microbiology 128, 26932698.

Haupt, I., Fricke, H., Cerna, J. \& Rychlik, I. (1976). Effect of the leucomycin-like macrolide antibiotic turimycin on ribosomal peptidyltransferase from Escherichia coli. Journal of Antibiotics 29, 1314-1319.

Khokhlov, A. S., Anisova, L. N., Tovarova, I. I., Kleiner, E. M., Kovalenko, I. V., Krasilnikova, O. I., Kornitskaya, E. Y. \& Pliner, S. A. (1973). Effect of A-factor on the growth of asporogenous mutants of Streptomyces griseus, not producing this factor. Zeitschrift für allgemeine Mikrobiologie 13, 647-655.

MCCann, P. A. \& Pogell, B. M. (1979). Panamycin : a new antibiotic and stimulator of aerial mycelia formation. Journal of Antibiotics 32, 673-678.

NaKano, M. M. \& Ogawara, H. (1980). Multiple effects induced by unstable mutation in Streptomyces lavendulae. Journal of Antibiotics 33, 420-425.

Rautenstein, Y. I., Solovyeva, N. Y., Roth, M., Noack, D. \& Klaus, S. (1976). Lysogeny of the culture of Streptomyces hygroscopicus producing antibiotic turimycin and some characteristics of its temperate phage. Mikrobiologiva 45, 922-925.

Redshaw, P. A., McCann, P. A., Pentella, M. A. \& Pogell, B. M. (1979). Simultaneous loss of multiple differentiated functions in aerial mycelium-negative isolates of streptomycetes. Journal of Bacteriology 137, 891-899.

Roth, M. \& NoACK, D. (1982). Genetic stability of differentiated functions in Streptomyces hygroscopicus in relation to conditions of continuous culture. Journal of General Microbiology 128, 107-114.

Roth, M., Noack, D., Solovyeva, N. Y. \& RautenSTEIN, Y. I. (1978). Effective enrichment of the temperate actinophage $\mathrm{SH} 3$ and its interaction with Streptomyces hygroscopicus. Zeitschrift für allgemeine Mikrobiologie 18, 147-149. 